\title{
NEW EXPERIMENTAL RESULTS WITH OPTICAL DIFFRACTION RADIATION DIAGNOSTICS*
}

\author{
E. Chiadroni ${ }^{\dagger}$, M. Castellano, LNF-INFN, Frascati, Italy \\ A. Cianchi, INFN Roma "Tor Vergata”, Roma, Italy \\ K. Honkavaara, G. Kube, DESY, Hamburg, Germany
}

\section{Abstract}

The characterization of the transverse phase space for high charge density and high energy electron beams is demanding for the successful development of the next generation light sources and linear colliders.

The interest in a non-invasive and non-intercepting beam diagnostics is increasingly high due to the stringent features of such beams. Optical Diffraction Radiation (ODR) is considered as one of the most promising candidates to measure the transverse beam size and angular divergence, i.e. the transverse emittance.

An experiment, based on the detection of the ODR angular distribution, has been set up at DESY FLASH Facility to measure the electron beam transverse parameters. In this paper we report the preliminary analysis on the incoherent diffraction radiation produced by a $900 \mathrm{MeV}$ energy electron beam going through a $0.5 \mathrm{~mm}$ rectangular slit.

\section{INTRODUCTION}

The final goal of our experiment is measuring the transverse beam size and divergence. A dedicate analysis of the Optical Diffraction Radiation (ODR) angular distribution [1] allows then to separate the two effects. If the beam is in a waist on the DR screen, the transverse emittance can be derived with a single non-intercepting measurement.

Some modifications have been done on the hardware in order to let synchrotron radiation (SR) contribute as less as possible. At this regard, a thin metallic screen (Fig. 2) has been installed to stop SR, but during measurements it has shown interesting peculiarities which need to be further investigated, both analytically and experimentally.

\section{DR THEORY}

DR is produced when a charged particle goes through a slit or passes by the edge of a metallic screen, due to the interaction between the EM field of the traveling charge and the target surface [2]. The intensity of the radiation increases linearly with charge and is proportional to $e^{-\frac{2 \pi a}{\gamma \lambda}}$, where $a$ is the slit aperture, $\gamma$ the Lorentz factor and $\lambda$ the emitted wavelength. The factor $\frac{\gamma \lambda}{2 \pi}$, called as DR impact parameter, is the natural size of the radial extension of the EM field, thus when $a \cong \frac{\gamma \lambda}{2 \pi} \mathrm{DR}$ is emitted.

\footnotetext{
* Work supported by the European Community Infra-structure Activity under the FP6 "Structuring the European Research Area" program (CARE, contract number RII3-CT-2003-506395)

$\dagger$ enrica.chiadroni@lnf.infn.it
}

The DR angular distribution is produced by the interference of radiation from both edges of the slit. The visibility of the interference fringes is mainly affected by beam parameters in the plane orthogonal to the slit aperture: when the transverse beam size is increased, both the peak intensity and the central minimum increase, resulting in the reduction of their ratio. The effect is also affected, in a slightly different way, by the angular divergence of the beam: the ODR angular distribution becomes wider and the intensity of the minimum higher, when the beam divergence increases.

\section{EXPERIMENTAL APPARATUS}

Our experiment is carried out at FLASH, Free electron LASer in Hamburg, at DESY. FLASH is an excellent facility for this experiment, since it can drive long bunch trains, up to 800 bunches per macropulse allowing a high charge operation, and it has a good long term stability, a small transverse emittance ( $\sim 2 \mathrm{~mm} \mathrm{mrad})$, and a high electron beam energy, approaching $1 \mathrm{GeV}$.

Our experimental set-up is placed in the by-pass beam line (Fig. 1) very far (about $40 \mathrm{~m}$ ) from the dipole magnets in order to minimize the contribution coming from the synchrotron light.

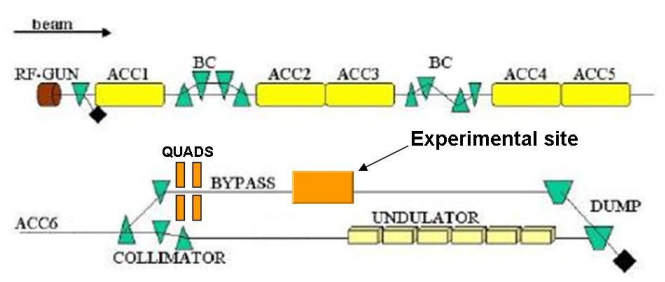

Figure 1: FLASH layout and experimental site.

The experimental apparatus has an aluminized silicon nitride screen (DR screen) mounted at $45^{\circ}$ angle with respect to the beam direction. The DR screen is constructed by lithographic technique starting from a silicon nitride wafer and opening two slits, one of $0.5 \mathrm{~mm}$ and the other of 1 $\mathrm{mm}$ aperture, by means of chemical etching. The slits are spaced by $2 \mathrm{~cm}$ and the space between the slits is used as a standard OTR screen. The main advantage of the silicon nitride with respect to $\mathrm{SiO}_{2}$ [3] is a much less etching rate which preserves the silicon substrate from damages and makes the surface much more uniform. An aluminum layer is deposited by sputtering on the target to enhance the reflectivity. 
A thin shielding mask (Fig. 2) is mounted at $45^{\circ}$ with respect to the DR target and normally to the direction of beam propagation. Two slits, $1 \mathrm{~mm}$ and $2 \mathrm{~mm}$ aperture, are machined in order to let both the beam and the radiation field go through without interacting with the screen. Furthermore the screen surface is machined such that reflections should be suppressed. The great advantage of this screen is the strong minimization of the SR background. On the other end, interference effects between the ODR emitted on the shielding mask in the forward direction and the radiation from the DR target are observed in the ODR angular distribution pattern (see next section).

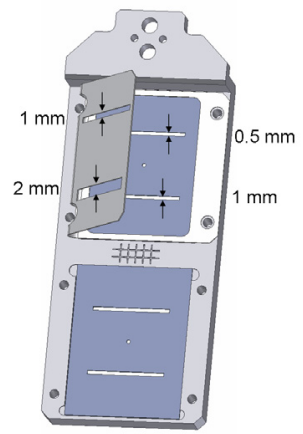

Figure 2: Sketch of the shielding mask. The DR radiator is visible behind.

Radiation from the target is reflected by a mirror and sent through an optical system to the camera. Two lenses, one to image the beam, the other one to produce the DR angular distribution, can be selected. They have different focal length in order to have the focus on the same plane. Two interferential filters, at $800 \mathrm{~nm}$ and $450 \mathrm{~nm}$, and a polarizer may be inserted on the optical axis. Due to the very low radiation intensity, a high sensitivity CCD camera (Hamamatsu Model C4742-98-LGLAG2) is used. The optical system layout is shown in Fig. 3.

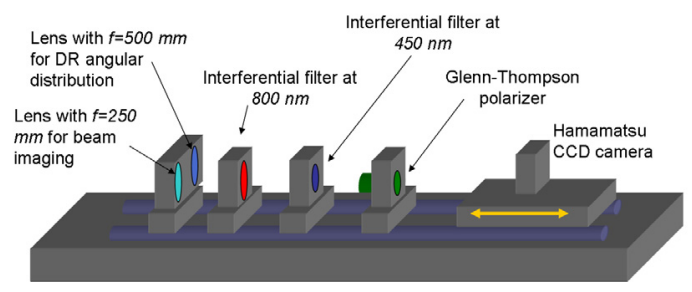

Figure 3: Sketch of the optical system.

\section{PRELIMINARY RESULTS}

To reduce the impact of SR background two big improvements have been done, on one side on the hardware with the installation of the shield screen and, on the other side, on the electron beam optics with runtime entirely dedicated to optimize the beam transport on the by-pass. Moreover the higher energy and the better quality of the beam allowed to get promising results. Unfortunately a severe contribution of the dark current (Fig. 4) from the cathode was a big drawback, being from time to time a source of transition radiation (see Fig. 7c).

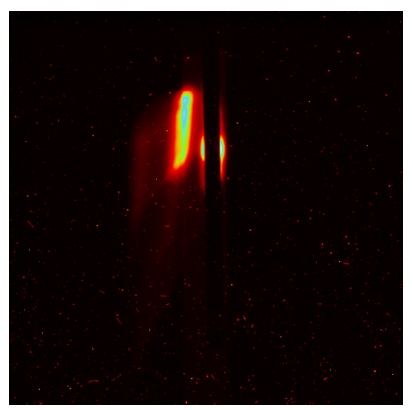

Figure 4: Beam in the $0.5 \mathrm{~mm}$ slit. On the left edge side the severe contribution of the dark current is visible.

In this section we report the preliminary results obtained with a $900 \mathrm{MeV}$ electron beam energy going through a $0.5 \mathrm{~mm}$ slit. During measurements reported in this paper, FLASH was operated with up to 11 electron bunches $(1 \mathrm{nC}$ per bunch) per macropulse with $1 \mathrm{MHz}$ bunch spacing. The macropulse repetition rate was $5 \mathrm{~Hz}$.

The image of the beam and its intensity projection are shown in Fig. 5a and Fig. 5b, respectively. The rms beam transverse size, evaluated from a Gaussian fit, is about $80 \mu \mathrm{m}$.

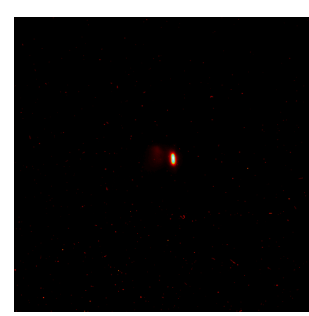

(a) Beam image

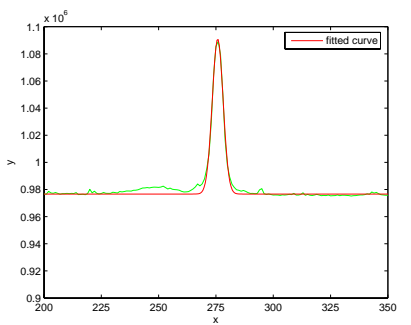

(b) Beam projection
Figure 5: Image of the beam on the OTR screen (a) and the projection fit (b).

The analysis of the Optical Transition Radiation (OTR) angular distribution (Fig. 6) allowed an estimation of the beam energy of about $820 \mathrm{MeV}$.

\section{Measurements}

The first part of our shifts has been dedicated to the optimization of the beam transport, in terms of both small rms transverse size and low gradient in the quadrupoles in order to avoid any contribution of synchrotron light coming from them. The strong contribution of the dark current (Fig. 7c) could not be avoided, but easily subtracted because it does not depend on the beam.

In this conditions several scans have been done moving the 


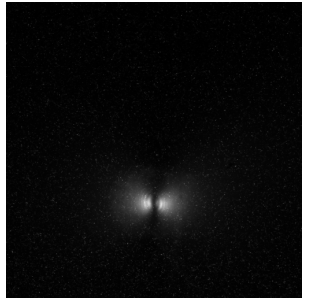

(a) OTR angular distribution

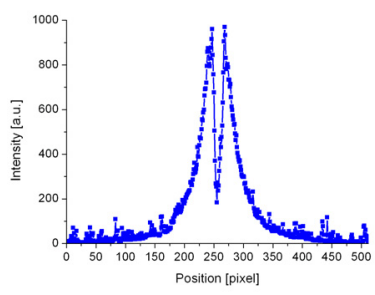

(b) OTR profile
Figure 6: OTR angular distribution with polarizer and 800 $\mathrm{nm}$ filter: a) image and b) projection of central line.

beam transversely in the slit and looking at the ODR angular distribution. Figure 7a) shows the ODR angular distribution in the case the beam goes through the $0.5 \mathrm{~mm}$ slit with an impact parameter of $150 \mu \mathrm{m}$. It is worth noting

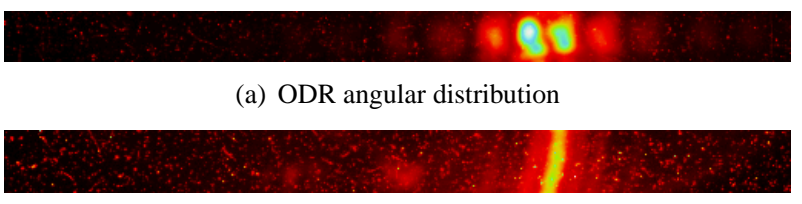

(b) SR background

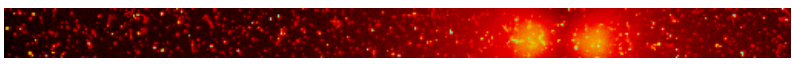

(c) Dark current background

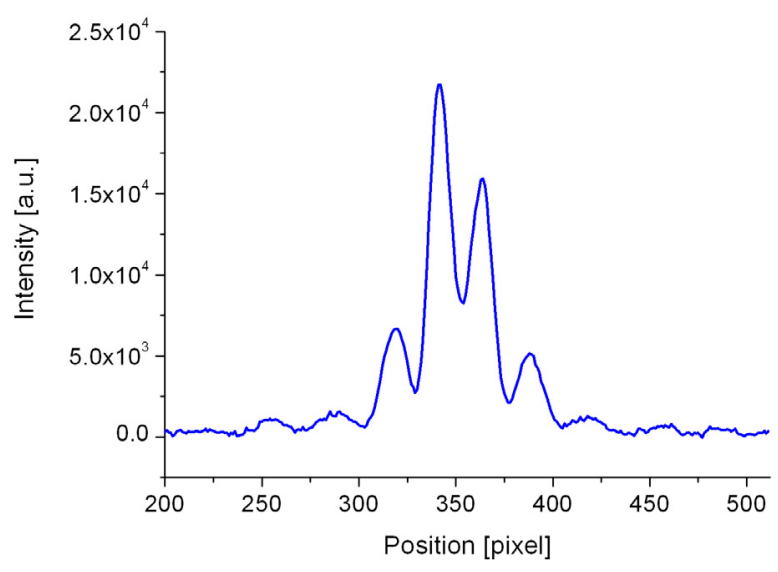

(d) ODR projection

Figure 7: ODR angular distribution (a), SR background, dark current-generated background (c) and vertical projection (d). Both $800 \mathrm{~nm}$ interference filter and polarizer are inserted.

the clear evidence of the higher orders of oscillation, highlighted by the interference effects between the two ODR sources, the one produced on the shielding mask in the forward direction (FDR), and the one generated by the DR screen in the backward direction (BDR). Since the amplitude of the two sources are opposite in sign, the interference results in the supression of the central peaks and the enhancement of the side maxima. Figure 8 shows the ODR angular distribution for a complete scan done moving the slit edge transversely in order to change the impact parameter. The asymmetry with respect to the central minimum must be addicted to the fact that the two slits (the one on the shielding mask and the one on the DR target) are not perfectly aligned, resulting in a different impact parameter.

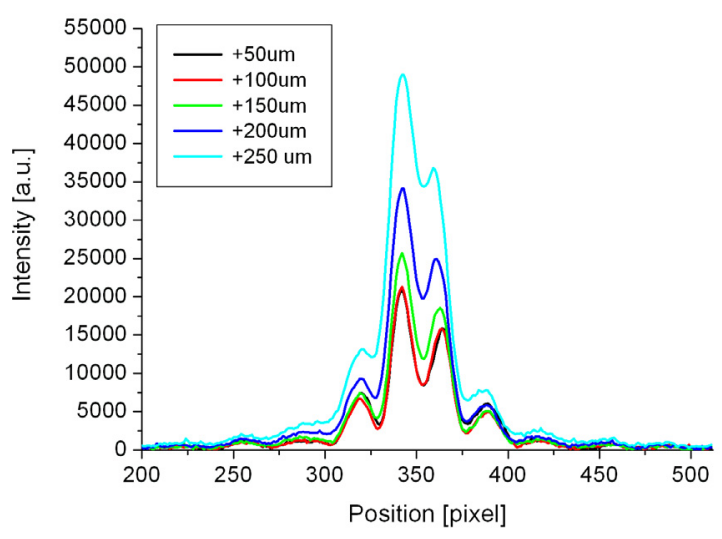

Figure 8: Angular distributions for different positions of the beam with respect to the center of the slit. Both the polarizer and the $800 \mathrm{~nm}$ filter are inserted.

\section{CONCLUSIONS}

The installation of a shielding mask produced interesting results. On one side it has helped in the reduction of the background signal coming from SR, on the other side it has highlighted the lateral peaks of the ODR angular distribution. A detailed and quantitative study of these effects is ongoing.

\section{ACKNOWLEDGEMENT}

Special thanks are to FLASH shift crews, in particular, the authors are grateful for Nina Golubeva and Vladimir Balandin for beam optics calculations and tuning the linac in order to get optimum beam conditions required for the experiment. We thank also R. Sorchetti and L. Cacciotti (INFN-LNF) for their fundamental technical assitance.

\section{REFERENCES}

[1] M. Castellano, Nucl. Instr. and Meth. in Phys. Res. A 394, 275 (1997)

[2] M.L. Ter-Mikaelian, "High-Energy Electromagnetic Processes in Condensed Media", Wiley-Interscience

[3] E. Cianci et al., "Micromachined silicon slit for beam diagnostic in particle accelerator", Proc. SPIE, 4557, pp.242-249 (2001)

[4] E. Chiadroni et al., Proceedings of PAC07, Albuquerque, New Mexico, USA 